\title{
Evaluation de la qualité des eaux de puits à usage domestique dans les quartiers défavorisés de quatre communes d'Abidjan (Côte d'Ivoire) : Koumassi, Marcory, Port-Bouet et Treichville
}

\author{
Ossey Bernard YAPO ${ }^{1 *}$, Véronique MAMBO ${ }^{1}$, Arsène SEKA ${ }^{1}$, Marie Jeanne Adélaïde \\ OHOU $^{1}$, Félix KONAN ${ }^{2}$, Valérie GOUZILE ${ }^{1}$, Abiba Sanogo TIDOU ${ }^{1}$, \\ Kouamé Victor KOUAME ${ }^{1}$ et Pascal HOUENOU ${ }^{1}$
}

\author{
${ }^{1}$ Laboratoire des Sciences de l'Environnement (L.S.E.), UFR-SGE, Université d'Abobo-Adjamé, 02 B.P. 801 \\ Abidjan 02, Côte d'Ivoire, tel: 0022520304309, fax: 0022520304256. \\ ${ }^{2}$ Laboratoire d'Environnement et Biologie Aquatique (LEBA), UFR-SGE, Université d'Abobo-Adjamé, O2 B.P. \\ 801 Abidjan 02, Côte d'Ivoire, tel: 0022523469475, fax: 0022520304256 \\ *Auteur correspondant, E-mail : yapossey@yahoo.fr; Tel: 0022507530260
}

\section{RESUME}

La qualité des eaux de puits à usages domestiques dans les quartiers précaires de quatre communes de la ville d'Abidjan a été étudiée à travers l'analyse des paramètres physico-chimiques et microbiologiques couramment utilisés pour la qualité de l'eau de consommation. Les concentrations en nitrates varient entre 0 et $286 \mathrm{mg} / \mathrm{L}$ et celles de l'ammonium entre 3,6 et $39,6 \mathrm{mg} / \mathrm{L}$. Plus de $80 \%$ des puits ont des teneurs en nitrates supérieures à la norme de $50 \mathrm{mg} / \mathrm{L}$ requise, ce qui traduit que les eaux sont d'une mauvaise qualité pour la boisson, mais subissent l'impact d'une insalubrité urbaine. En plus, la qualité des eaux de puits est variable et dépend d'un certain nombre de facteurs tels que l'emplacement des puits par rapport aux sources de contamination urbaines endogènes. La dégradation de la qualité des eaux est fortement impactée par le défaut d'assainissement des quartiers précaires. L'interprétation des données d'analyse, la corrélation existante entre les eaux de puits et la répartition des eaux de puits en groupes sont réalisées en utilisant l'Analyse en Composante Principale Normée (ACPN). Plusieurs facteurs conditionnent la contamination des eaux de puits par les polluants minéraux tels que $\mathrm{NO}_{3}{ }^{-}, \mathrm{NH}_{4}{ }^{+}, \mathrm{SO}_{4}{ }^{2-}$ et $\mathrm{Cl}^{-}$. Le défaut d'assainissement dans ces quartiers, la mauvaise gestion des déchets urbains, la faible profondeur de la nappe, la nature des sols et la perméabilité de l'aquifère exploité sont les preuves de la vulnérabilité des eaux des puits. En effet, les résultats acquis font ressortir la forte influence de l'activité urbaine sur la qualité de ces eaux qui sont fortement chargées en ions nitrates et ammonium, signe d'une pollution urbaine.

(c) 2010 International Formulae Group. All rights reserved.

Mots clés : Pollution, eaux de puits, assainissement, analyse en composante principale normée, sels dissous.

\section{INTRODUCTION}

L'accès à l'eau potable et à l'assainissement représentent un combat quotidien pour des centaines de milliers de citadins qui vivent principalement dans les grandes villes des pays en développement (Hinrischen Don et al., 2002; UNWater/ WWAP, 2006). La croissance démographique considérable que connaissent les pays en développement et leurs conditions 
économiques difficiles entraînent une urbanisation anarchique difficilement contrôlable (Chippaux et al., 2002), notamment dans les villes, source de la dégradation de la qualité physico-chimique et microbiologique des eaux de surface et souterraines.

Abidjan, capitale économique de la Côte d'Ivoire, connaît une détérioration de la qualité de ses eaux de surface et souterraines due à une urbanisation anarchique et mal maîtrisée. Les besoins en eau des populations sont grandissants et l'urbanisation incontrôlée pourrait exacerber les impacts négatifs sur la qualité des ressources en eaux. En effet, l'approvisionnement en eau potable de la population abidjanaise est uniquement assuré par les eaux souterraines contenues dans les formations sableuses (Kouamé, 2007). Bien que régi par un système d'adduction, avec l'implantation de la Société de Distribution d'Eau de Côte d'Ivoire (SODECI), qui couvre grandement le service en eau potable, certaines populations, notamment des quartiers défavorisés, ont toujours recours à l'eau des puits traditionnels, qui constituent des sources alternatives d'approvisionnement en eau.

Ces quartiers ne disposent pas non plus d'un assainissement de base, et comme partout ailleurs, dans ces types de quartiers, l'on note, selon Coulibaly et al. (2004) et Dongo et al. (2008), la présence de nombreuses maladies hydriques (Yonkeu et al., 2003 ; Granado et al., 2006 ; Mpakan et al., 2006).

En effet, les puits sont exposés à des risques de contamination par des polluants chimiques, biologiques ou microbiologiques générés par les activités urbaines. De nombreuses études y ont été conduites, précisant les différents états physicochimiques des eaux souterraines en Côte d'Ivoire. Elles ont mis en évidence une importante pollution par les nitrates (Fofana, 2005 ; Traoré, 2005 ; Savané et al., 2005), notamment dans les eaux de la nappe profonde où de fortes teneurs en nitrates jusqu'à $200 \mathrm{mg} . \mathrm{L}^{-1}$ ont été observées dans les eaux de forage (Faillat, 1990), systématiquement situés dans ou aux abords immédiats de villages à habitat concentré, le plus souvent d'extension ou d'implantation récente. Ces études se sont moins intéressées aux puits traditionnels, alimentés par la nappe superficielle; comprise entre 0 et $10 \mathrm{~m}$ de profondeur (Kouamé, 2009). La présente étude qui s'inscrit à la suite de ces travaux, se propose, d'une part, de caractériser la qualité des eaux de puits à usages domestiques dans les quartiers précaires de quatre communes de la ville d'Abidjan (Koumassi, Marcory, PortBouet et Treichville), et, d'autre part, de faire l'inventaire des causes de pollution d'origine naturelle et anthropique, et d'évaluer l'impact de cette pollution sur la qualité des eaux souterraines dans la zone d'étude sur la base des procédés analytiques et statistiques.

\section{MATERIEL ET METHODES}

\section{Présentation de la zone d'étude}

Le District d'Abidjan, situé au sud de la Côte d'Ivoire (Figure 1), couvre une superficie de $2119 \mathrm{Km}^{2}$, et regroupe une population estimée à environ 3125890 habitants en 1998 (INS, 2001). Cette population s'est accrue aujourd'hui d'une manière considérable pour atteindre les 5 millions d'habitants en 2006 du fait de la crise sociopolitique que connaît la Côte d'Ivoire depuis septembre 2002 (MPD, 2006). Abidjan est située sur le cordon littoral entre l'océan atlantique et la lagune Ebrié. Située à une altitude de $20 \mathrm{~m}$, Abidjan est soumise à un climat caractérisé par deux saisons de pluie alternées par deux saisons sèches. Son bassin sédimentaire côtier est constitué des aquifères $\mathrm{du}$ quaternaire et du continental terminal (Angui et Biemi, 1984). La nappe d'eau souterraine est considérée comme libre, bien que captive à certains endroits.

A l'instar des grandes villes africaines, Abidjan connaît une démographie galopante avec une urbanisation mal contrôlée. Cette situation favorise l'émergence de quartiers anarchiques périphériques contrastant avec le 


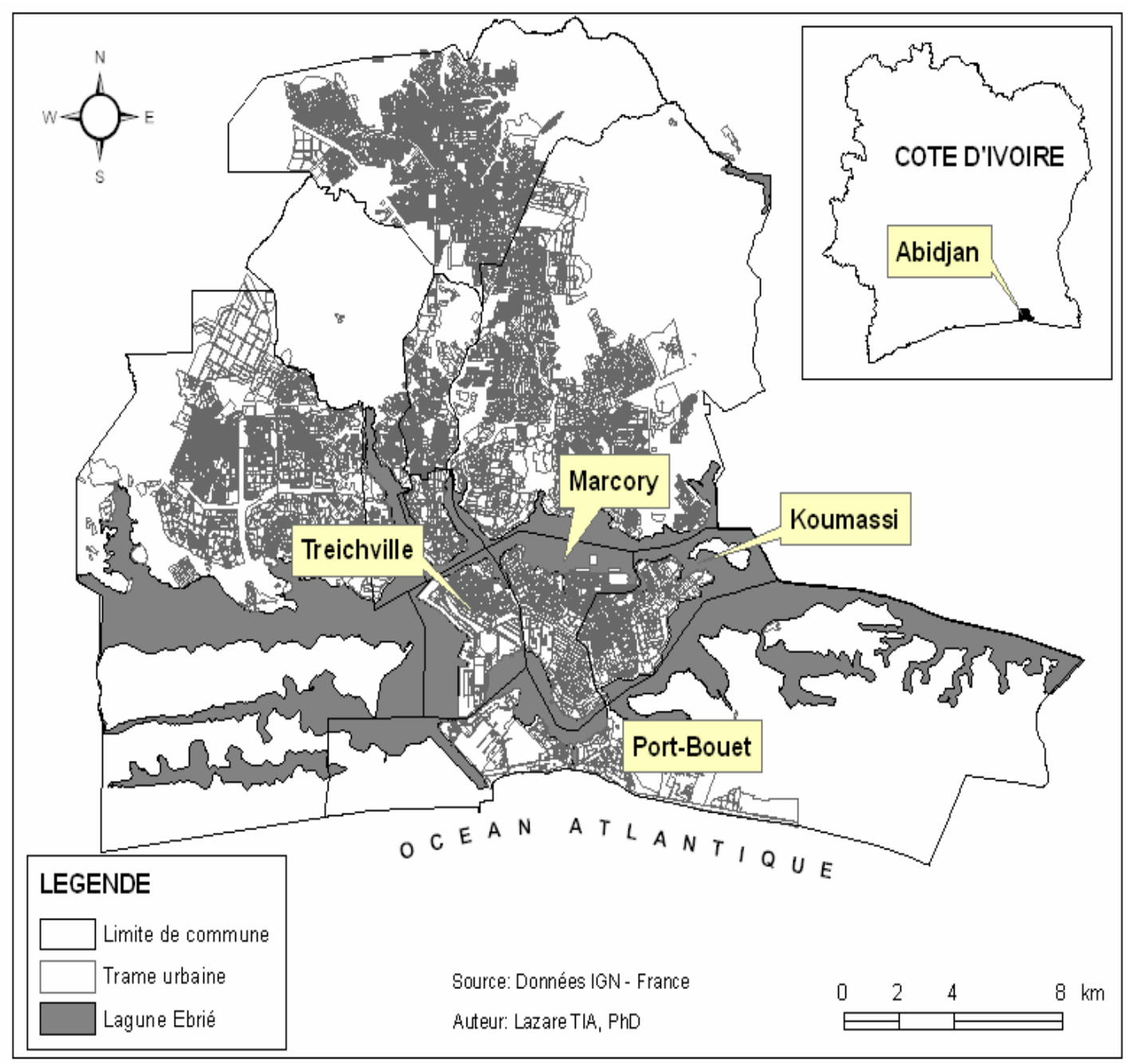

Figure 1: Localisation géographique de la zone d'étude et des localités prospectées du district d'Abidjan : Port-Bouet plus proche de l'océan atlantique est limité au nord par Koumassi, Marcory et Treichville; Koumassi est limité à l'ouest par Marcory, Marcory est limité à l'ouest par Treichville, ces quatre communes partagent en commun la lagune Ebrié.

centre ville. Ces quartiers d'habitations précaires sont installés sur des sites qui manquent d'hygiène, de systèmes d'assainissement et souvent d'infrastructures sanitaires et sociales. Selon le Bureau National d'Etudes Techniques et de Développement (BNETD, 1992), Abidjan compte 75 quartiers précaires. Notre étude a porté sur 22 quartiers au sud d'Abidjan, dont 12 à Port-Bouet, 6 à Koumassi, 3 à Marcory et 1 à Treichville. Ces 22 quartiers occupent une superficie totale d'environ 323 hectares. Le mode d'habitation diffère en taille et en configuration, les maisons d'habitation sont réparties en 53100 ménages soit 6 à 8 personnes par ménage (BNETD, 1992).

L'inefficience des services d'approvisionnement en eau et d'assainissement dans ces quartiers précaires est due entre autres à l'occupation anarchique des sols, à l'extrême pauvreté des populations et à l'insuffisance des moyens financiers de la 
part des pouvoirs publics (UN-Habitat, 2001).

\section{Echantillonnage}

\section{Choix des sites de prélèvement}

Une enquête préliminaire a été menée à l'aide de questionnaire et de fiches d'inspection sanitaire dans les quatre communes de Treichville, Koumassi, Marcory et Koumassi. Elle s'est déroulée auprès des populations utilisatrices des eaux des puits et des centres sanitaires des quartiers précaires étudiés. Les observations et questions ont porté sur le type d'habitat, le système d'évacuation des excréta, l'évaluation de la protection des puits contre les sources de pollutions, les risques sanitaires, l'emploi et le niveau de revenu dans les ménages, le mode d'approvisionnement et les diverses utilisations de l'eau.

Les systèmes de protection des puits sont constitués de margelle, de fermeture et de revêtement interne ou cuvelage. A Treichville, $60 \%$ des puits ont une margelle, contre $40 \%$ à Port-Bouet, $30 \%$ à Koumassi et $25 \%$ à Marcory. Les puits sont souvent à ciel ouvert, seulement 5\% sont fermés à Port-Bouet, 10\% à Koumassi, $15 \%$ à Marcory et $20 \%$ à Treichville. On note néanmoins que plus de $50 \%$ des puits possèdent un revêtement interne. Ces revêtements sont constitués de matériaux en ciment (environ 60\%), de pneus usés $(25 \%)$, de matériaux en bois et divers (10\%). Sur la base des résultats de l'enquête réalisée, il ressort que les puits sont dans un environnement insalubre.

La protection des puits est défaillante dans la majorité des cas avec une absence de système de drainage. Les eaux de ces puits sont donc exposées à une pollution de surface, mais à des contaminations par infiltration.

Deux types de puits réalisés par la population elle-même ont été identifiés:

- les puisards traditionnels temporaires dont la profondeur est généralement inférieure à 10 mètres et le diamètre variant entre 0,8 et 2 mètres, avec une hauteur d'eau inférieure à 0,5 mètre.
- les puits pérennes, pouvant atteindre 20 mètres de profondeur, des diamètres de 0,8 à $1 \mathrm{~m}$ et un soutènement des parois assuré par des pierres, des briques ou des barriques métalliques (en fer).

\section{Prélèvement d'échantillons des eaux de puits}

Le choix des puits à prélever a été fixé à la lumière d'une étude des paramètres physico-chimiques, lors de l'enquête préliminaire. La fréquence d'utilisation des puits par les populations, la proximité des sources de pollution pour les eaux souterraines sont les critères qui nous ont guidés dans le choix des puits. Au total, 24 puits ont été échantillonnés pour caractériser l'impact des rejets polluants urbains sur la qualité physico-chimique et microbiologique des eaux de puits à usages domestiques, à raison de 6 puits par zone d'étude (Koumassi, Port-Bouet, Marcory et Treichville).

Le prélèvement, le transport et la conservation des échantillons font référence au protocole défini par l'Agence Française de Normalisation (AFNOR (NF-T 90-10). Ainsi, les échantillons d'eaux de puits ont été recueillis dans des bouteilles de 1 litre en polyéthylène, préalablement lavées. Chaque bouteille est rincée trois fois avec l'eau à prélever, puis rempli à refus et fermée hermétiquement avant d'être portée dans la glacière.

Les prélèvements pour les analyses microbiologiques ont été effectués à l'aide de bouteilles préalablement autoclavées. Les échantillons ont été conservés à $4{ }^{\circ} \mathrm{C}$ dans des glacières pendant le transport au laboratoire, puis ont été analysés dans les 24 heures qui suivent.

Pour chaque prélèvement, 14 paramètres ont été mesurés dont 11 liés à la physico-chimie et 3 paramètres microbiologiques. La température, la conductivité électrique, les Sels Dissous Totaux (TDS) et le pH ont été mesurés sur le terrain. 


\section{Méthodes d'analyses}

$\mathrm{Au}$ laboratoire, les ions majeurs $\left(\mathrm{Cl}^{-}\right.$, $\mathrm{SO}_{4}^{-}$et $\mathrm{HCO}_{3}{ }^{-}$), la dureté total $(\mathrm{TH})$ et les sels nutritifs ont été dosés. Les chlorures et l'alcalinité ont été dosés par titration (Rodier, 1996 ; AFNOR, 1997). Les ions $\mathrm{SO}_{4}{ }^{2-}$ ont été dosés par néphélométrie. Les dérivés azotés par contre, ont été mesurés par colorimétrie à l'aide d'un spectrophotomètre Hach DR 2010, les nitrates $\left(\mathrm{NO}_{3}{ }^{-}\right)$par réduction au cadmium, les nitrites $\left(\mathrm{NO}_{2}{ }^{-}\right)$par diazotation et l'ammonium $\left(\mathrm{NH}_{4}^{+}\right)$par la méthode à l'indophénol.

Les analyses microbiologiques ont permis d'identifier et de dénombrer les Streptocoques fécaux, les Coliformes totaux et les Clostridium sulfito-réducteurs. Ces microorganismes ont été identifiés et dénombrés en filtrant des aliquotes homogènes de $100 \mathrm{ml}$ sur une membrane dont le diamètre des pores est de $0,45 \mu \mathrm{m}$. Les membranes ont ensuite été placées sur des milieux sélectifs pendant 24 heures à $37^{\circ} \mathrm{C}$ à l'étuve thermo-statée. Les milieux suivants ont été utilisés: la gélose KF pour les Streptocoques fécaux, le coli ID pour les coliformes totaux, la gélose TSN (Tryptone Sulfite Néomycine) pour le Clostridium sulfito-réducteur dont le plus marquant est le Clostridium perfringens.

\section{Analyses statistiques}

Deux tests non-paramétriques ont été utilisés pour les comparaisons inter et intra des paramètres de la qualité des eaux des puits dans les quatre communes étudiées.

Le test de Kruskal-Wallis a été utilisé à un seuil de significativité de 95\% (p<0,05), pour mettre en évidence les éventuelles variations entre les différents paramètres de qualité des eaux de puits à l'intérieur de chacune des communes (variabilité intracommunale).

Le test de Mann-Whitney a été utilisé également pour un seuil de significativité de 95\% ( $\mathrm{p}<0,05)$, pour étudier la variabilité inter-communale de la qualité des eaux des puits. Les communes ont été comparées deux- à-deux, et l'évolution spatiale de chaque paramètre a été étudiée à l'aide des boîtes à moustaches ou "boxplot" (Tomassone et al., 1993).

L'étude de la typologie de la pollution des eaux de puits a été basée sur une Analyse en Composante Principale Normées (A.C.P.N). Les valeurs propres, les cartes factorielles et les cercles de corrélations ont été obtenus avec le logiciel XLStat 7.0 (Addinsoft SARL).

Il a permis de traiter des caractères numériques jouant tous le même rôle (Bourouche et Saporta, 1983).

\section{RESULTATS \\ Aspect qualitatif des eaux des puits}

L'eau souterraine n'est pas toujours pure puisqu'elle contient toujours des substances dont les concentrations et la stabilité dépendent des paramètres tels que le pH du milieu, la texture de sols (Banton et al., 1997), et surtout de l'état de salubrité de l'environnement. La Figure 2 présente la distribution des teneurs des différents paramètres pour l'analyse de la qualité des eaux des puits. Les puits sont disposés sur l'axe des abscisses dans le sens d'évolution nord-sud, selon le profil horizontal. Les communes de Treichville, Marcory, Koumassi et Port-Bouet sont situées au sud d'Abidjan, mais pris dans cet ordre, parcourent le sens nord-sud.

En effet, la carte piézométrique de la nappe d'Abidjan en régime permanent révèle que, les eaux souterraines du District d'Abidjan coulent dans la direction nord-sud, les lignes de courant sont orientées vers les cours d'eau de l'Agnéby et de la Mé, du fait que le niveau de l'eau est aussi faible (entre 0 et $0,2 \mathrm{~m}$ ). Les niveaux piézométriques oscillent entre $55 \mathrm{~m}$ au Nord et $1 \mathrm{~m}$ au Sud (Kouamé et al., 2009).

\section{Paramètres physiques}

La température montre une certaine stabilité spatiale, elles varient entre 24,6 et $32,0{ }^{\circ} \mathrm{C}$ avec une moyenne de $28,4( \pm 2,3){ }^{\circ} \mathrm{C}$ 
$\mathrm{pH}$

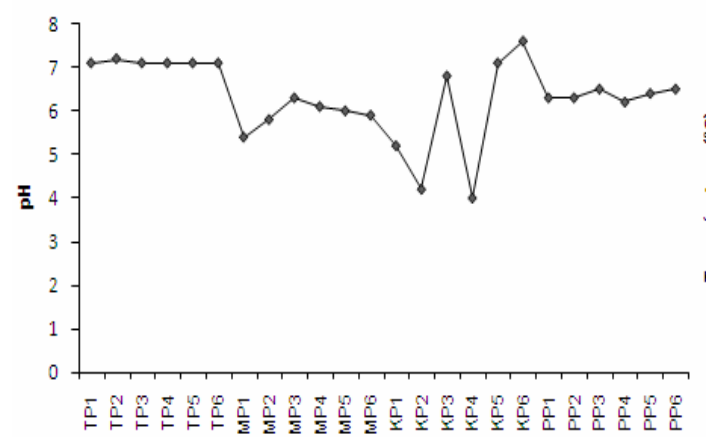

CND

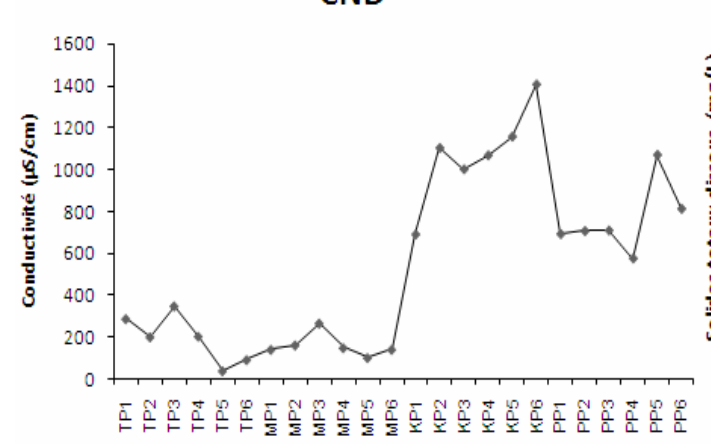

Temp

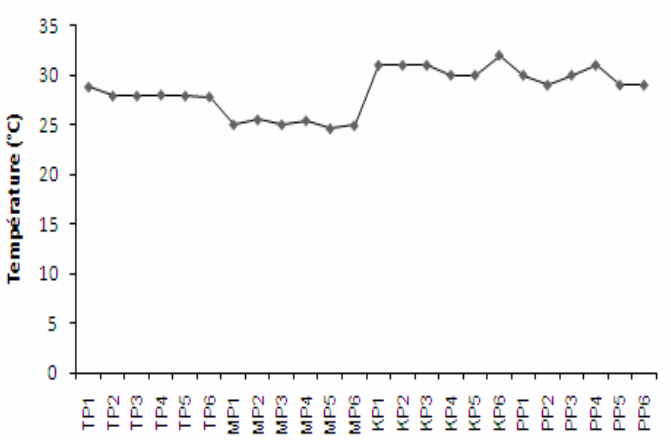

TDS

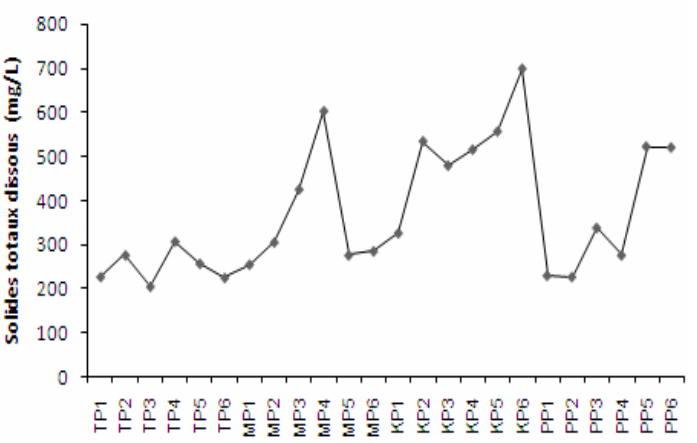

Figure 2 : Evolution spatiale des valeurs des principaux paramètres physiques indicateurs de la qualité des eaux des puits de Treichville (TP), Marcory (MP), Koumassi (KP) et Port-Bouet (PP). Temp = température, $T D S=$ Sels dissous totaux $C N D=$ conductivité électrique .

pour l'ensemble des eaux de puits (Figure 2). Les $\mathrm{pH}$ varient de 4,0 à 7,6 avec une moyenne de $6,3( \pm 0,9)$, ils répondent aux normes de potabilité (Normes Françaises, 1999; WHO, 2008). Mais ici, on remarque que les eaux ont une tendance acide $(\mathrm{pH}<7)$. L'acidité est d'autant plus importante dans les puits de Koumassi. La conductivité et le TDS montrent une large variation de la composition chimique des eaux. La conductivité varie entre un minimum de $42,7 \mu \mathrm{S} / \mathrm{cm}$ et un maximum de $1406,0 \mu \mathrm{S} / \mathrm{cm}$. On constate une minéralisation croissante nord-sud allant de Treichville à Port-Bouet. La charge saline totale dissoute des eaux souterraines (TDS) est comprise entre 205 et $698 \mathrm{mg} / \mathrm{L}$ avec une valeur moyenne de $369,4 \mathrm{mg} / \mathrm{L}$ confirmant ainsi l'ordre de grandeur de la conductivité des eaux.

\section{Paramètres chimiques}

Les concentrations des paramètres chimiques sont variables et ne présentent pas de stabilité spatiale (Figure 3). Les concentrations d'ammonium sont supérieures à $0,5 \mathrm{mg} / \mathrm{L}$, les concentrations naturelles dans les eaux souterraines et de surface sont généralement inférieures à $0,2 \mathrm{mg} / \mathrm{L}$. Les eaux souterraines anaérobies peuvent en contenir 

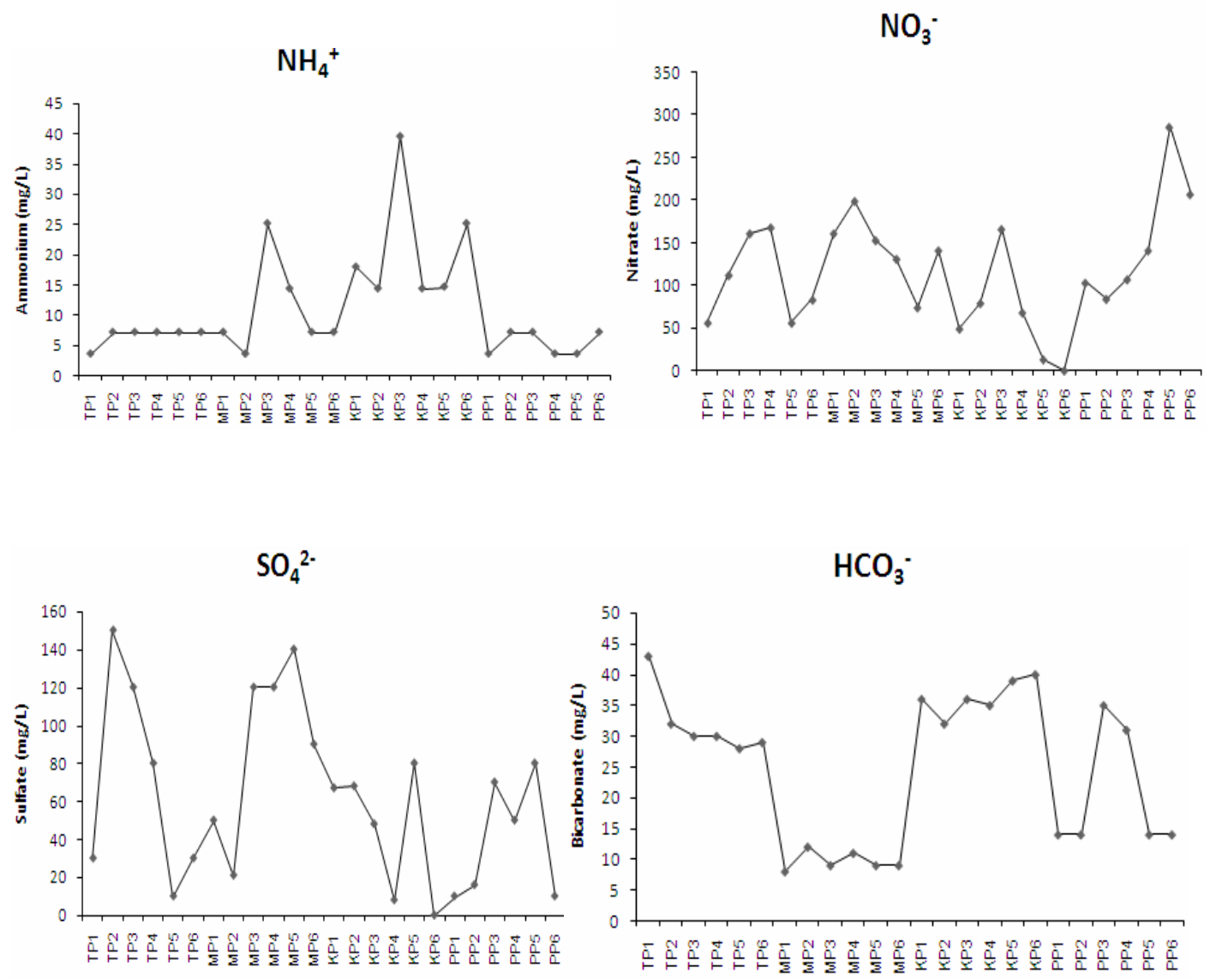

$\mathrm{TH}$

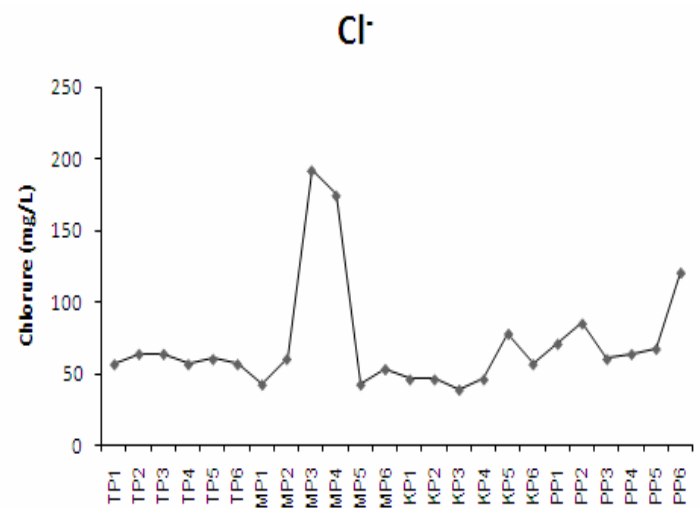

Figure 3 : Evolution spatiale des valeurs des principaux paramètres Chimiques indicateurs de la qualité des eaux des puits de Treichville (TP), Marcory (MP), Koumassi (KP) et Port-Bouet (PP). $\mathrm{TH}=$ Titre Hydrotimétrique, $\mathrm{HCO}_{3}^{-}=$ion bicarbonate, $\mathrm{Cl}=$ ion Chlorue, $\mathrm{NO}_{3}^{-}=$ion nitrate, $\mathrm{NH}_{4}^{+}=$ion ammonium, $\mathrm{SO}_{4}{ }^{2}$ = ion sulfate. 
jusqu'à 3 mg/L (OMS, 2007). Les concentrations des nitrates sont supérieures à $50 \mathrm{mg} / \mathrm{L}$ dans plus de $80 \%$ des puits. En effet, 21 puits sur 24 échantillonnés, ont une concentration en nitrate supérieure à $50 \mathrm{mg} / \mathrm{L}$. Les espèces azotées $\left(\mathrm{NH}_{4}^{+}, \mathrm{NO}_{2}^{-}\right.$et surtout $\mathrm{NO}_{3}{ }^{-}$) étant de bons indicateurs d'une dégradation de la qualité de l'eau, les eaux des puits étudiés ne peuvent servir de consommation humaine, tant les concentrations d'ammonium et de nitrate sont élevées, atteignant des extrêmes de $39 \mathrm{mg} / \mathrm{L}$ pour l'ammonium à Koumassi et $286 \mathrm{mg} / \mathrm{L}$ pour les nitrates à Port-Bouet. De façon générale, leurs concentrations sont largement supérieures aux normes admises pour les eaux de consommation humaine qui sont de 0,5 $\mathrm{mg} / \mathrm{L}$ pour l'ammonium et $50 \mathrm{mg} / \mathrm{L}$ pour les nitrates (WHO, 2003; 2008). En effet, les aquifères de cette zone ne sont plus exploités pour l'alimentation en eau potable de la population abidjanaise par la SODECI, compte tenu du taux élevé de nitrate observé dans certains forages mais surtout à cause de l'intrusion saline (Kouadio et al., 1998; Jourda et al., 2006).

Les ions sulfates varient de 0 à 150 $\mathrm{mg} / \mathrm{L}$, et sont plus importants dans les puits de Treichville et de Marcory. On observe un gradient d'évolution nord-sud décroissant lorsqu'on se déplace de Treichville vers PortBouet. Les concentrations moyennes varient entre 39,3 $( \pm 31,5) \mathrm{mg} / \mathrm{L}$ à 90,2 $( \pm 46,2) \mathrm{mg} / \mathrm{L}$ et les écart-types observés traduisent l'hétérogénéité des mesures.

Dans les eaux souterraines, le pH et la spéciation des carbonates sont interdépendants et tributaires des bases fortes résultant de la dissociation des minéraux carbonatés et silicatés (Banton et al., 1997). Les ions $\mathrm{HCO}_{3}{ }^{-}$ constituent le constituant essentiel de l'alcalinité des eaux naturelles pour lesquelles le $\mathrm{pH}$ est proche de la neutralité. L'alcalinité varie à l'inverse des ions $\mathrm{H}^{+}$dans l'intervalle de $\mathrm{pH}$ compris entre 6 et 8 (Bobée et al., 1982).

Les concentrations en bicarbonates varient de $8 \mathrm{mg} / \mathrm{L}$ à $43 \mathrm{mg} / \mathrm{L}$, les valeurs les plus faibles étant mesurées à Marcory et les plus fortes à Treichville et Koumassi. Les valeurs du Titre Hydrotimétrique (TH ou dureté exprimée en $\mathrm{mg} / \mathrm{L}$ de $\mathrm{CaCO}_{3}$ ) n'excédant pas $500 \mathrm{mg} / \mathrm{L}$ sont jugées acceptables pour l'eau de boisson (WHO, 2008). En effet, ce paramètre traduit la capacité des eaux à réagir avec les savons ; une eau dure exigeant beaucoup plus de savon pour produire de la mousse. Cette dureté n'est pas liée à une substance unique, mais à divers ions métalliques polyvalents, principalement le calcium et le magnésium, bien que d'autres cations comme le baryum, le fer, le manganèse, le strontium et le zinc $y$ contribuent également. Sa valeur varie entre $50 \mathrm{mg} / \mathrm{L}$ et $258 \mathrm{mg} / \mathrm{L}$. On observe un gradient d'évolution croissant nord-sud lorsqu'on se déplace de Treichville vers Port-Bouet (Figure 3). La valeur moyenne des ions chlorures varient de 52,1 $( \pm 13,9) \mathrm{mg} / \mathrm{L}$ à $94,3( \pm 69,5)$ $\mathrm{mg} / \mathrm{L}$, avec des pics observés dans les puits de Marcory où l'on atteint la valeur maximale de $192 \mathrm{mg} / \mathrm{L}$.

\section{Paramètres microbiologiques}

Les charges moyennes des Clostrodium, des coliformes et streptocoques sont exprimées dans le Tableau 1 où l'on note une forte charge bactérienne des eaux. Les Clostridium sont plus importants à Koumassi, les coliformes et les streptocoques le sont plus à Treichville.

On note que parmi ces trois espèces microbiologiques, par ordre croissant d'importance, on a les Clostridium, les coliformes et les streptocoques dans les eaux des quatre communes étudiées. La qualité microbiologique des eaux montre qu'elles sont impropres à toute consommation avant traitement. En effet, l'eau destinée à la consommation et aux besoins des ménages ne doit pas contenir de micro-organismes pathogènes, aucun échantillon de $100 \mathrm{ml}$ d'une eau destinée à la consommation ne doit contenir de Clostridium, de coliformes et de streptocoques (WHO, 2008). 
Tableau 1: Charges moyennes microbiologiques dans eaux de puits des communes de Koumassi, Marcory, Port-Bouet et Treichville.

\begin{tabular}{lllll}
\hline \multirow{2}{*}{ Charges en UFC/100 ml } & \multicolumn{4}{c}{ Zones d'études } \\
\cline { 2 - 5 } & Koumassi & Marcory & Port-Bouet & Treichville \\
\hline Clostridium & 236 & 96 & 72 & 184 \\
Coliformes & 764 & 2000 & 1770 & 8167 \\
Streptocoques & 3702 & 1917 & 1142 & 33325 \\
\hline
\end{tabular}

\section{Variabilité spatiale des paramètres}

Pour vérifier la variabilité spatiale des paramètres physico-chimiques étudiés, il a donc été choisi le test d'hypothèse non paramétrique de Kruskal et Wallis (Siegel, 1956 ; Harris et al., 1987), et celui de Mann Witney pour les comparaisons inter et intra sujets. Il est réalisé sur les données classées, et a permis de vérifier l'appartenance ou pas des échantillons à une même population, eu égard aux moyennes, aux seuils classiques de $1 \%$ ou même 5\%. Les résultats de ces tests ont permis de construire les Figures 4 et 5 qui décrivent les variations spatiales des paramètres physico-chimiques de qualité des eaux de puits à l'intérieur de chacune des communes. Le test de Kruskal Wallis réalisé indique qu'il n'y a pas de différence significative dans la variation des ions chlorures, nitrates, sulfates et la dureté totale pour la caractérisation de la qualité des eaux de puits des localités étudiées. Par contre, il existe des différences statistiquement significatives entre la conductivité, les ions bicarbonates, le $\mathrm{pH}$, le taux de solide dissous et de la température pour lesquelles $\mathrm{p}<0,05$.

En effet, le test de Mann-Whitney combiné aux boîtes à moustaches, révèle que les valeurs de la conductivité, des ions bicarbonates et de la température des eaux des puits de Koumassi et de Port-Bouet sont plus élevées que celles de Marcory (Figure $5\left(\mathrm{~d}_{5}\right)$ ). Les puits de Koumassi, comparés à ceux de Treichville, présentent également des valeurs de conductivité, de température, de TDS et des teneurs en ammonium plus élevées.

Pour le $\mathrm{pH}$, les puits de Treichville ont des valeurs plus élevées que ceux de Port-
Bouet, puis de Marcory et Koumassi (Figure 4 (d4)). La valeur médiane obtenue à Marcory est néanmoins plus faible que celle de PortBouet et de Koumassi. L'on note que seuls les puits de Treichville présentent une valeur médiane de $\mathrm{pH}$ proche de la neutralité. Dans les trois autres communes, (Koumassi, PortBouet, Marcory), les $\mathrm{pH}$ des puits sont en dessous de 6,5; donc à tendance acide. L'acidité des eaux est plus importante dans les eaux des puits de Koumassi et Marcory où le pH atteint 4.

La comparaison des puits des localités de Koumassi et de Port-Bouet par le test de Mann-Whitney ( $\mathrm{p}<0,05)$ montre que les puits de Koumassi ont des températures et des concentrations en bicarbonate et en ammoniac plus élevées que celles de Port-Bouet.

Sur l'ensemble des paramètres utilisés pour ces comparaisons, seule la température des eaux des puits diffère entre toutes les localités. Cette différence peut s'expliquer par la période d'échantillonnage ou par la profondeur des puits.

\section{Analyse en composante principale de toutes les eaux}

Pour déterminer l'origine des concentrations observées et pour mieux évaluer l'effet de l'environnement urbain sur la qualité des eaux de puits, nous avons utilisé une méthode statistique dite Analyse en Composante Principale Normée (A.C.P.N) qui permet de transformer les variables quantitatives initiales, toutes plus corrélées entre elles, en nouvelles variables quantitatives, non corrélées, appelées 

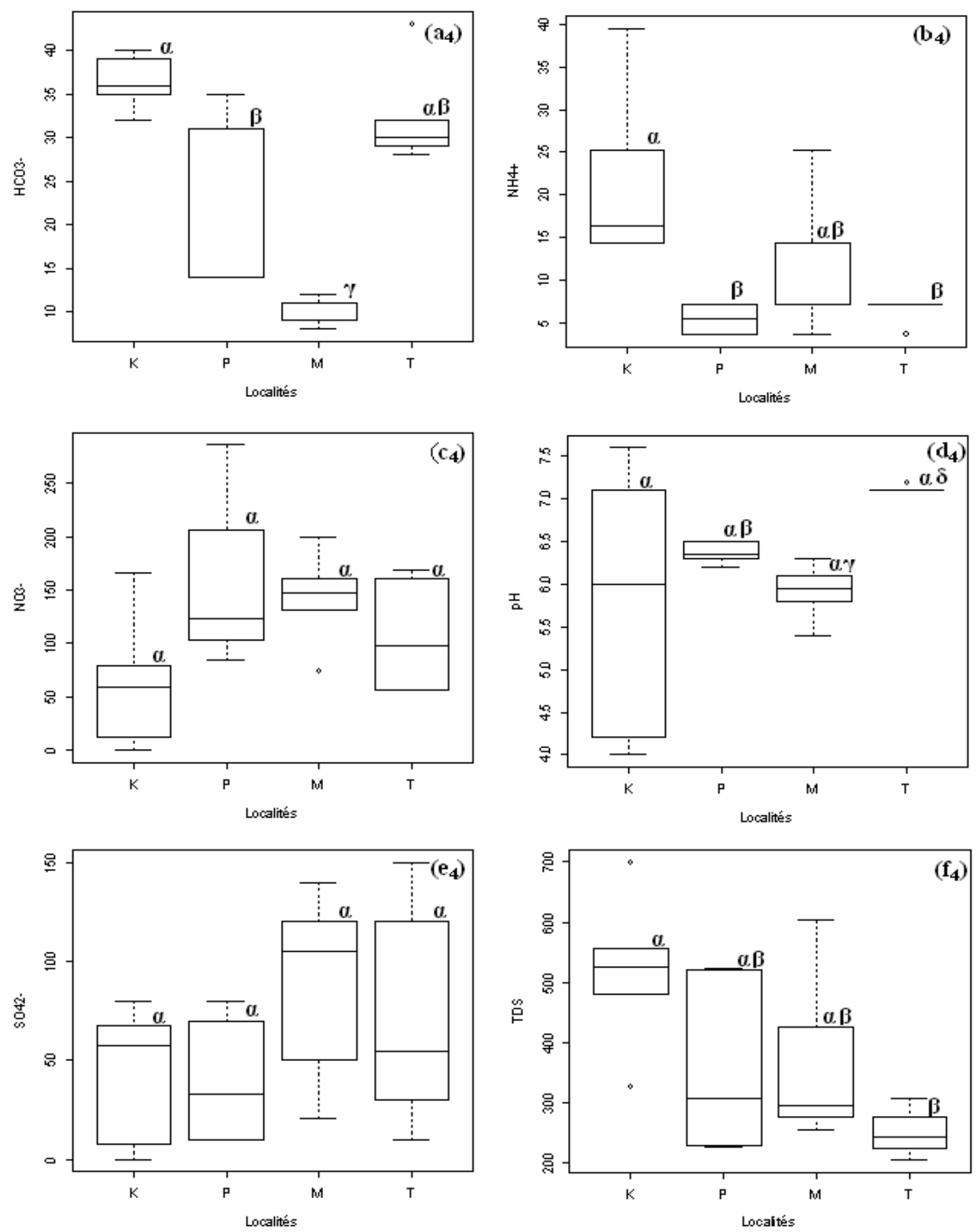

Figure 4: Boîtes de box de $\left(\mathrm{a}_{4}\right)$ l'alcalinité exprimée en $\mathrm{HCO}_{3}^{-},\left(\mathrm{b}_{4}\right)$ ion ammonium $\mathrm{NH}_{4}{ }^{+}$; $\left(\mathrm{c}_{4}\right)$ ions nitrate $\mathrm{NO}_{3}{ }^{-} ;\left(\mathrm{d}_{4}\right) \mathrm{pH} ;\left(\mathrm{e}_{4}\right)$ ions sulfates $\mathrm{SO}_{4}{ }^{2-} ;\left(\mathrm{f}_{4}\right)$ Taux de Sels Dissous TDS; des eaux de puits des communes de Koumassi (K), Port-Bouet (P), Marcory (M), Treichville (T). Pour un paramètre donné, il n'y a pas de différence significative entre deux boites portant une même lettre de l'alphabet grec $(\alpha, \beta, \gamma, \delta)$. Deux lettres différentes traduisent une variabilité importante entre deux points de prélèvement. Le seuil de significativité du test de Mann-Whitney est de $5 \%$.. 

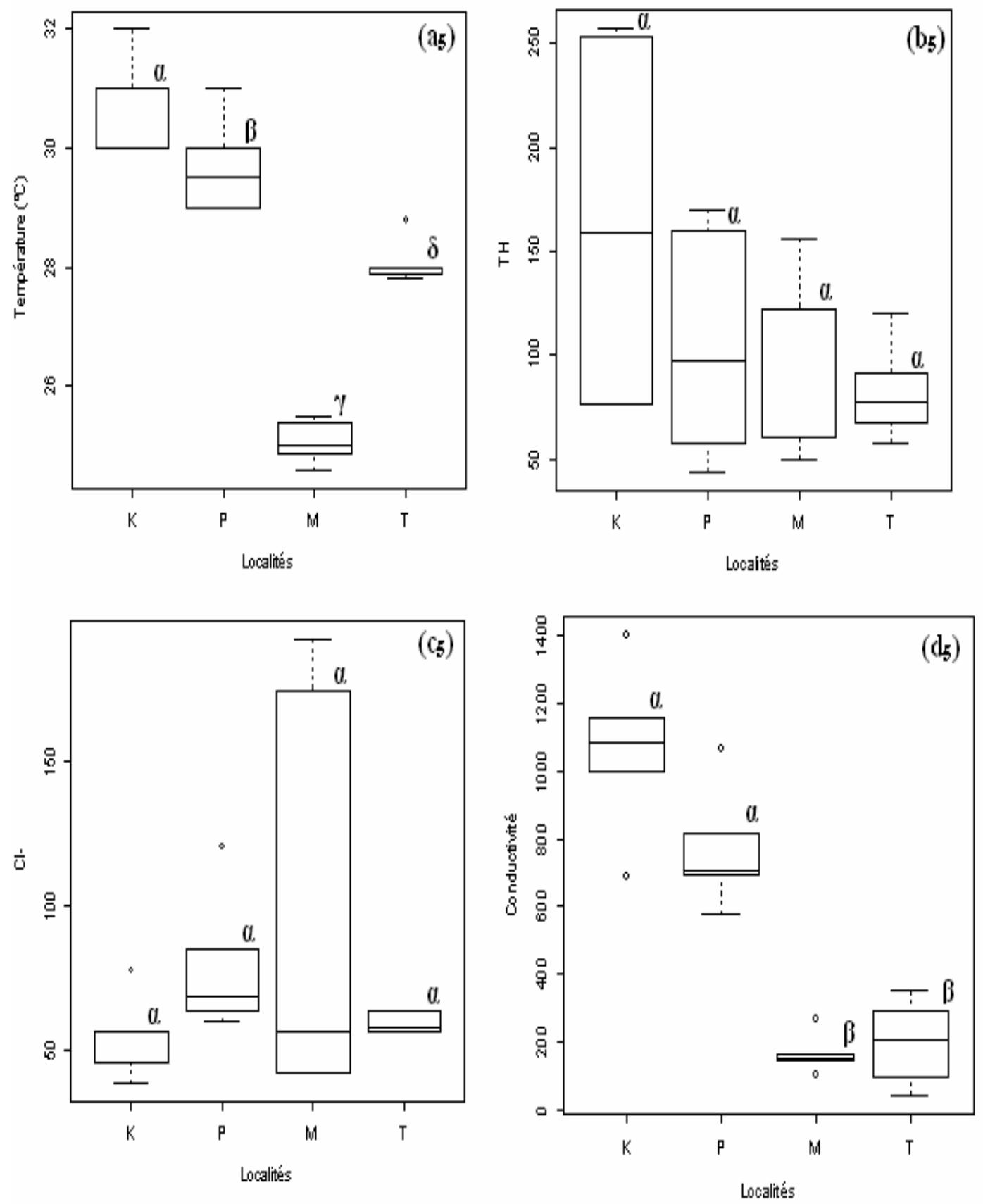

Figure 5: Boîtes de box de $\left(\mathrm{a}_{5}\right)$ Température ; $\left(\mathrm{b}_{5}\right)$ Titre Hydrotrimétrique Total THT ; $\left(\mathrm{c}_{5}\right)$ Chlorures $\mathrm{Cl}^{-}$; $\left(\mathrm{d}_{5}\right)$ Conductivité; des eaux de puits des communes de Koumassi (K), Port-Bouet (P), Marcory (M), Treichville $(\mathrm{T})$. Pour un paramètre donné, il n'y a pas de différence significative entre deux boîtes portant une même lettre de l'alphabet grec $(\alpha, \beta, \gamma, \delta)$. Deux lettres différentes traduisent une variabilité importante entre deux points de prélèvement. Le seuil de significativité du test de Mann-Whitney est de 5\%.. 
composantes principales (Davis, 1984). C'est une méthodologie largement utilisée pour interpréter les données hydrochimiques (Domenico et Schwartz, 1990; El AmraniPaaza et al., 1994 ; El Asslouj et al., 2007).

L'ACP est construite à partir des corrélations linéaires sur les variables centrées réduites, et la matrice des corrélations ayant servi à cette construction (Tableau 2). En gras sont représentées les valeurs significatives (hors diagonale) au seuil alpha $=0,05$ et pour un nombre d'individu $\mathrm{n}=24$. Les variations de la minéralisation des eaux sont régies par les teneurs en ammonium $(r=0,56)$, en calcium et/ou en magnésium $(r=0,55)$. La température est bien corrélée à la conductivité. Par exemple, les eaux des puits de Koumassi sont plus chaudes et plus salines. En effet, plus les puits sont profonds, plus la température des eaux s'élève. Une élévation de la température des eaux souterraines pourrait donc favoriser les échanges de sels minéraux entre l'eau et le sol, mais aussi favoriser l'intrusion des eaux salines dans une zone plus proche de la lagune Ebrié. La bonne corrélation entre la température, le bicarbonate $(r=0,77)$ pourrait traduire une acidification ou une dégradation du pouvoir tampon en cours, des eaux de puits, causée par l'intrusion de particules acides dont la température est l'élément catalyseur. Ce que traduit la corrélation entre le $\mathrm{pH}$ et les ions $\mathrm{HCO}_{3}{ }^{-}$.

Le traitement statistique a été poussé jusqu'à trois axes factoriels (F1, F2 et F3) qui expriment respectivement $35,9,16,7$ et $14 \%$ de la variance totale du nuage de points, soit une inertie cumulée des trois axes de $66,6 \%$ de l'information totale (Figure $6 \mathrm{~B}$ et $6 \mathrm{E}$ ).

D'une façon générale, l'axe d'inertie principal $\mathrm{F} 1$ exprime $35,9 \%$ de la variance. Il est défini positivement par la conductivité, la température, les ions bicarbonates $\left(\mathrm{HCO}_{3}{ }^{-}\right)$, TDS, TH et les ions $\mathrm{NH}_{4}^{+}$(Figure 6C). Cet axe peut être assimilé au caractère minéral des eaux défini par la conductivité, TDS et $\mathrm{HCO}_{3}{ }^{-}$.

L'axe d'inertie F2 exprime 16,7\% de la variance (Figure 6A). Il est défini positivement par $\mathrm{SO}_{4}{ }^{2-}$, TDS, mais légèrement par $\mathrm{NO}_{3}^{-}, \mathrm{Cl}^{-}$et $\mathrm{TH}$ (Figure 6C). Cet axe peut être assimilé à un gradient de pollution par les ions $\mathrm{NO}_{3}{ }^{-}, \mathrm{Cl}^{-}$et $\mathrm{SO}_{4}{ }^{2-}$, donc à la pollution des eaux des puits par les activités humaines.

L'axe F3 exprime $14 \%$ de la variance (Figure 6, B et E). Il est défini positivement avec le $\mathrm{pH}$, les ions $\mathrm{Cl}^{-}$et $\mathrm{NO}_{3}{ }^{-}$mais négativement avec $\mathrm{NH}_{4}{ }^{+}$(Figure $6 \mathrm{~F}$ ). Il peut être assimilé à un gradient de pollution par les activités humaines ayant un impact sur le $\mathrm{pH}$ des eaux.

Les cercles de corrélation formés par les axes $\mathrm{F} 1$ et $\mathrm{F} 2$ donnant $52,6 \%$ de l'information totale (Figure 6B), montre selon l'axe F1 (Figure 6C), une opposition entre les eaux riches en $\mathrm{NO}_{3}{ }^{-}, \mathrm{SO}_{4}{ }^{2-}$ et $\mathrm{Cl}^{-}$et les eaux pauvres en ces ions.

Selon F2, on observe une opposition entre les eaux fortement minéralisées par $\mathrm{NH}_{4}{ }^{+}$et $\mathrm{Ca}^{2+}$ et/ou $\mathrm{Mg}^{2+}\left(\mathrm{TH}=\left[\mathrm{Ca}^{2+}\right]+\right.$ $\left.\left[\mathrm{Mg}^{2+}\right]\right)$, et les eaux faiblement minéralisés. Il définit aussi une pollution croissante de gauche à droite due à des conditions de diminution de l'oxygène et donc à une augmentation des concentrations en ions $\mathrm{NH}_{4}^{+}$.

Dans le plan factoriel F1xF3, on retrouve à peu près la même configuration que F1xF2, sauf que selon F3, on observe une grande contribution des ions $\mathrm{Cl}^{-}$(anion de base faible) à l'élévation du $\mathrm{pH}$ et une augmentation des nitrates, s'accompagnent d'une augmentation des ions chlorures.

Ces trois axes permettent de subdiviser le plan en quatre groupes de puits qui diffèrent par leur qualité physico-chimiques (Figure 6 A et D)

- La classe 1 caractérise les puits dont les eaux sont fortement affectées par l'activité urbaine et donc peu minéralisées. Ces eaux ont des concentrations plus élevées en ions $\mathrm{NO}_{3}{ }^{-}, \mathrm{SO}_{4}{ }^{2-}$ et $\mathrm{Cl}^{-}$. Il s'agit des puits situés dans les zones de Marcory et Treichville (Figure 6, A et D).

- La classe 2 caractérise les puits dont les eaux sont fortement minéralisées et fortement affectées par l'activité urbaine. Ces 
Tableau 2: Matrice des corrélations entre les paramètres physico-chimiques des eaux.

\begin{tabular}{lllllllllll}
\hline & $\mathrm{pH}$ & $\mathrm{T}^{\circ} \mathrm{C}$ & $\mathrm{NO}_{3}{ }^{-}$ & $\mathrm{Cl}^{-}$ & $\mathrm{NH}_{4}{ }^{+}$ & $\mathrm{CND}$ & $\mathrm{SO}_{4}{ }^{2-}$ & $\mathrm{HCO}_{3}{ }^{-}$ & $\mathrm{THT}$ & $\mathrm{TDS}$ \\
\hline $\mathrm{pH}$ & 1 & & & & & & & & & \\
$\mathrm{~T}^{\circ} \mathrm{C}$ & 0,088 & 1 & & & & & & & & \\
$\mathrm{NO}_{3}{ }^{-}$ & $-0,088$ & $-0,306$ & 1 & & & & & & & \\
$\mathrm{Cl}^{-}$ & 0,274 & $-0,086$ & 0,213 & 1 & & & & & & \\
$\mathrm{NH}_{4}{ }^{+}$ & $-0,016$ & 0,239 & $-0,327$ & $-0,180$ & 1 & & & & & \\
$\mathrm{CND}^{2-}$ & 0,043 & $\mathbf{0 , 8 2 2}$ & $-0,126$ & 0,107 & 0,308 & 1 & & & & \\
$\mathrm{SO}_{4}{ }^{--}$ & 0,002 & $\mathbf{- 0 , 4 6 4}$ & 0,239 & 0,107 & 0,084 & $-0,282$ & 1 & & & \\
$\mathrm{HCO}_{3}^{-}$ & $\mathbf{0 , 4 0 9}$ & $\mathbf{0 , 7 6 7}$ & $\mathbf{- 0 , 5 1 0}$ & $-0,214$ & 0,279 & $\mathbf{0 , 5 8 1}$ & $-0,257$ & 1 & & \\
$\mathrm{THT}$ & 0,140 & $\mathbf{0 , 4 0 7}$ & $-0,047$ & $-0,030$ & 0,385 & $\mathbf{0 , 5 2 5}$ & 0,055 & 0,335 & 1 & \\
$\mathrm{TDS}$ & $-0,129$ & 0,376 & $-0,019$ & 0,084 & $\mathbf{0 , 5 5 9}$ & $\mathbf{0 , 5 8 7}$ & 0,032 & 0,210 & $\mathbf{0 , 5 5 4}$ & 1 \\
\hline
\end{tabular}

En gras, valeurs significatives (hors diagonale) au seuil alpha $=0,050$ (test bilatéral)

eaux ont une forte conductivité mais sont caractérisées par la présence d'ions ammonium. Elles sont le fait des puits de Port-Bouet et de Koumassi alimentés par une infiltration des eaux lagunaires.

- La classe 3 caractérise les puits dont les eaux sont faiblement minéralisées et faiblement affectées par l'activité urbaine. Elles sont caractéristiques des puits des zones de Marcory.

- La classe 4 caractérise les eaux fortement minéralisées et moins affectées par l'activité urbaine. Ce sont les eaux des puits de Port-Bouet et la majorité des puits de Koumassi (Figure 6, A et D).

Du point de vue qualitatif, les eaux des puits des classes 3 et 4 peuvent convenir à un usage domestique après désinfection.

\section{DISCUSSION}

Le défaut d'assainissement observé dans les quartiers précaires affecte négativement la qualité physicochimique et surtout microbiologique des eaux de puits. L'insalubrité urbaine qui caractérise l'environnement des puits, enrichit les eaux en ammonium et en nitrates. Il s'agit notamment des puits de la zone de Koumassi, de PortBouet, Marcory moins les eaux des puits de
Treichville. Ce que confirment d'ailleurs, les résultats des analyses microbiologiques qui traduisent une forte contamination de ces eaux par des germes d'origine fécale. En effet, au plan sanitaire, $60 \%$ des puits ont une margelle de protection à Treichville contre $40 \%$ à PortBouet, $30 \%$ à Koumassi et $25 \%$ à Marcory. Le mode d'évacuation des excréta est dominé par l'utilisation des fosses. Ainsi $90 \%$ et $95 \%$ des populations, respectivement à Koumassi et Marcory, utilisent des latrines à fosses contre $40 \%$ et $30 \%$ respectivement à Port-Bouet et Treichville. Ces pratiques sont des sources d'enrichissement et de contamination des eaux souterraines par les ions nitrates, ammonium et chlorures. Nos résultats confirment la pollution générale des nappes superficielles d'Abidjan comprise entre 0 et $10 \mathrm{~m}$ de profondeur. La pollution bactériologique est largement confirmée dans la plupart des puits. En effet, les caractéristiques microbiologiques des eaux des puits étudiés traduisent bien une contamination des eaux par des germes d'origine fécale. Les agents contaminateurs de ces eaux proviennent du tube digestif de l'homme ou de l'animal et sont éliminés principalement par les matières fécales, éventuellement par les urines (Hasley et Leclerc, 1993). 

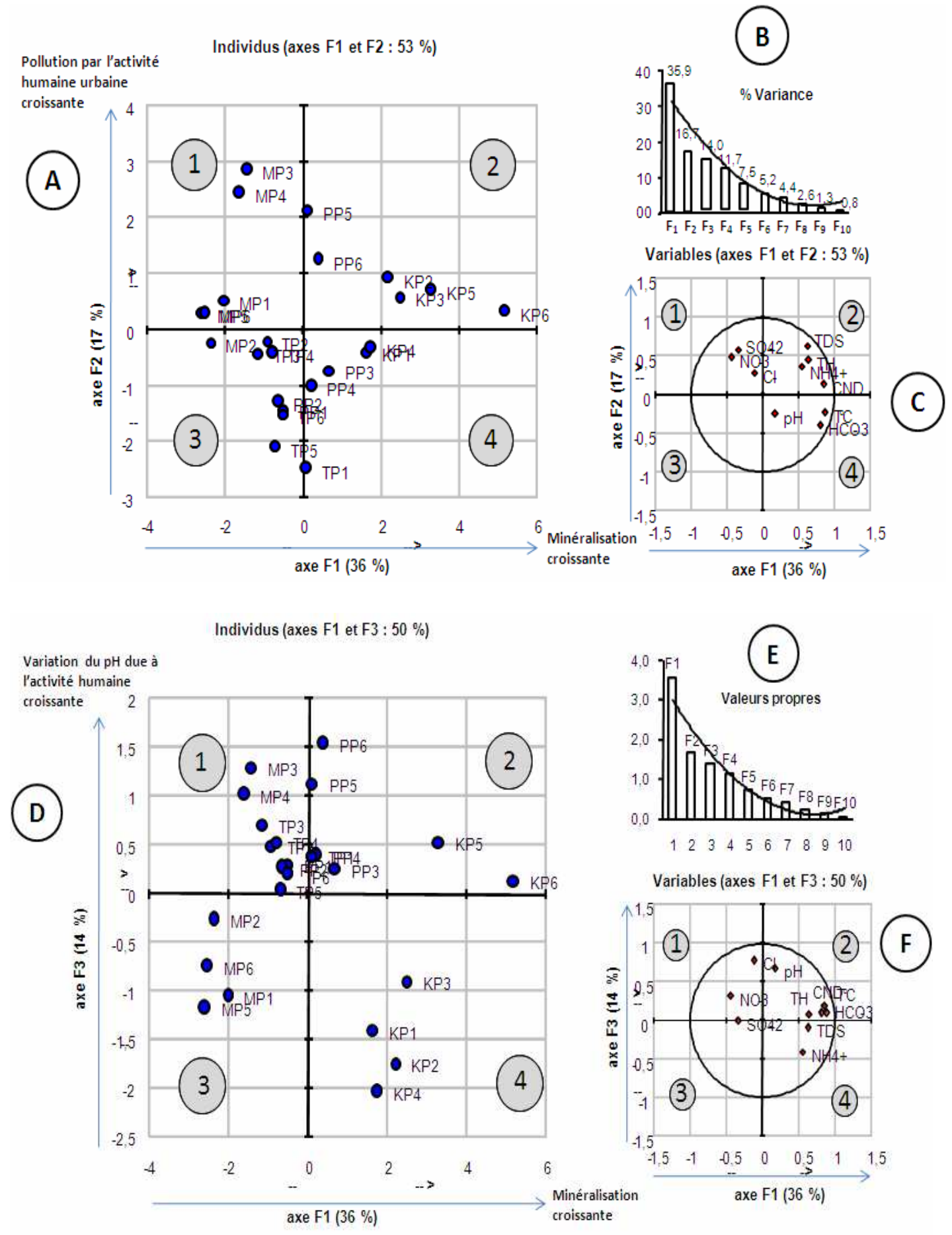

Figure 6: Représentation graphique dans les plans $\mathrm{F} 1 \mathrm{xF} 2$ et $\mathrm{F} 1 \mathrm{xF} 3$ de l'analyse en composantes principales normées des eaux de puits des localités de Koumassi (K), Port-Bouet (P), Marcory (M), Treichville (T); A et D : cartes factoriels des puits ; C et F : cartes factorielles des variables ; B et $\mathrm{E}:$ pourcentage d'inertie des composantes principales. 
Les mesures de conductivité électrique, de concentration en chlorures, en nitrates, en ammonium et en sulfates montrent également que la pollution touche la nappe phréatique superficielle d'Abidjan qui alimente les puits traditionnels à usage domestique. La présence d'azote ammoniacal est le signe de la contamination des eaux des puits par des apports exogènes, mais aussi traduit l'absence d'un processus de dégradation complète de la matière organique (Rodier et al., 1996). En accord avec Chippaux et al. (2002), les facteurs de pollution découlent bien des problèmes majeurs liés au défaut d'assainissement des quartiers précaires et tiennent essentiellement au manque de salubrité. Ils sont liés à l'absence de dispositif d'assainissement approprié à la protection des eaux souterraines et au défaut de collecte des ordures ménagères. En matière d'assainissement individuel, les équipements correspondent dans la majorité des cas à des latrines et puisards très mal entretenus et proches de la nappe. La gestion des ordures ménagères est rudimentaire, avec des dépotoirs sauvages constitués sur les voies ravinées par le ruissellement des pluies.

$\mathrm{Si}$ les origines potentielles de la pollution des puits sont bien identifiées, il reste cependant d'en comprendre les mécanismes de transfert. Selon Kouamé et al. (2009), il suffit de 1 mois pour que $70 \%$ des eaux infiltrées atteignent la nappe phréatique superficielle située entre 0 et $10 \mathrm{~m}$. La qualité des eaux des puits dépend ainsi de la nature de son sous-sol, mais subit l'impact des activités humaines, avec l'infiltration des eaux usées domestiques.

Enfin, la pollution par l'azote doit être soulignée. Une origine géologique est à exclure. Cette pollution azotée provient vraisemblablement des matières organiques humaines et animales infiltrées. La forte contamination d'origine fécale s'accompagne naturellement d'une pollution azotée que l'on voit à travers les fortes concentrations d'ammonium et de nitrate.

Quant aux ions $\mathrm{Cl}^{-}$et $\mathrm{SO}_{4}{ }^{2-}$, ils peuvent avoir une origine naturelle et anthropique, notamment pour les puits des quartiers de Koumassi et ceux qui sont situés à proximité des eaux lagunaires. Les sulfates peuvent provenir des roches sédimentaires, minerais, des transformations chimiques et biochimiques de $\mathrm{SO}_{2}, \mathrm{H}_{2} \mathrm{~S}$, mais selon Potvin et al. (1983), les sulfates que l'on retrouve dans les eaux sont généralement un bon indicateur du transport de polluants acides d'origine anthropique, puisqu'on les retrouve naturellement très peu dans les sols et soussols. Ainsi, les excès de concentrations supérieures à $100 \mathrm{mg} / \mathrm{L}$ peuvent être liés à des rejets d'eaux usées, à la présence des puits à proximité des décharges (dépôts sauvages), ou au lessivage du milieu urbain suite aux évènements pluvieux. Les eaux des puits sont donc exposées à des contaminations soit de nature chimique, soit de nature microbiologique générées par un environnement immédiat malsain. Le risque de pollution des eaux de la nappe par les ions $\mathrm{NO}_{3}{ }^{-}$et $\mathrm{NH}_{4}{ }^{+}$et aussi par $\mathrm{Al}^{3+}$, qui sont liés aux activités humaines, est accentué par la forte concentration de population dans ces quartiers, mais aussi par le faible raccordement au réseau d'assainissement collectif et individuel de la ville d'Abidjan (Soro, 2003). L'éventualité d'une pollution de la nappe profonde doit être envisagée sérieusement, surtout que les forages réalisés en nappe profonde sont utilisés pour l'adduction en eau potable de la ville d'Abidjan. C'est pourquoi, les études doivent se poursuivre pour la surveillance de l'évolution de pollution de la nappe, notamment en profondeur.

\section{Conclusion}

Les données recueillies au cours de cette étude ont permis de dresser un portrait de la qualité physico-chimique et 
microbiologique des eaux de puits à usages domestiques dans les quartiers précaires de Koumassi, Marcory, Port-Bouet et Treichville. Les eaux de certains puits échantillonnés sont de mauvaise qualité et ne satisfont pas aux normes de qualité de l'eau potable. La qualité physico-chimique des eaux de puits varie d'une commune à une autre, selon que les puits soient proches du front lagunaire ou des sources anthropiques de pollutions. La dispersion des valeurs des mesures témoigne en effet de sources de pollution ponctuelles existantes, même à l'intérieure de chaque zone. Ces fluctuations pourraient entre autres être liées aux différents facteurs endogènes tels que l'infiltration d'eaux usées, contamination par les eaux usées de fosses, l'intrusion d'eaux lagunaires, des lixiviats de dépôts d'ordures ménagères et de rejets industriels. Les nappes superficielles, accessibles par les puits sont contaminées par des matières organiques d'origine humaines, animales. Cette pollution trouve très probablement ses origines dans l'insuffisance des infrastructures d'assainissement et de collecte des ordures ménagères.

La profondeur de la nappe est donc un facteur déterminant, car il s'agit de la nappe superficielle dont les eaux sont très vulnérables à l'activité urbaine. Plus la nappe est profonde, plus elle est protégée contre une pollution urbaine diffuse. L'on pourrait toutefois s'inquiéter de la contamination de certains forages exploités par la SODECI par les nitrates. Ces inquiétudes sont déjà confirmées et l'éventualité d'une pollution bactériologique de la nappe profonde doit être envisagée sérieusement, surtout que cette ressource est exploitée systématiquement pour l'adduction en eau potable. Une ponction intense et régulière du contenu de la nappe profonde pourrait augmenter significativement la circulation des eaux souterraines, par appel d'eau de la nappe superficielle et d'eaux de surfaces, aggravant ainsi la contamination de la nappe profonde par les polluants de surface.
Les études doivent se poursuivre pour surveiller l'évolution de la pollution de la nappe superficielle et de la nappe profonde. Les modes de transfert de l'eau et des polluants dans la zone non saturée d'une part, dans la nappe d'autre part, doivent maintenant être impérativement caractérisés, afin de hiérarchiser les différents facteurs de pollution et prendre les mesures conservatoires appropriées.

\section{REFERENCES BIBLIOGRAPHIQUES}

Aghui N, Biémi J. 1984. Bassin sédimentaire de Côte d'Ivoire. Géologie et hydrogéologie des nappes de la région d'Abidjan et risques de pollution. Annales Université Abidjan, Série C (Sciences), 10 : 313-347.

AFNOR (Association Française de Normalisation). 1997. Qualité de l'Eau: Terminologie, Echantillonnage et Evaluation des Méthodes (Tome 1, 3 ème édn). AFNOR : Paris, France.

Banton O, Bangoy LM, Chevalier S, Houénou P, Lafrance P, Rivard Ch. 1997. Hydrogéologie Multiscience Environnementale des Eaux Souterraines. Presses de l'Université du Québec, AUPELF ; 460.

BNETD (Bureau National d'Etude Technique et Développement). 1992. Rapport sur les quartiers précaires d'habitat à Abidjan. BNETD ; 216 - 324.

Bouroche JM, Saporta G. 1992. L'analyse des Données (Collection Que sais-je ? $\mathrm{n}^{\circ}$ $854,5^{\text {ème }}$ édn). Presses Universitaires de France : Paris ; 1-27

Bobée B, Grimard Y, Lachance M, Tessier A. 1982. Nature et étendue de l'acidification des lacs du Québec. Rapport scientifique $\mathrm{n}^{\circ} 140,28-68$.

Chippaux JP, Houssier S, Gross P, Bouvier C, Brissaud F. 2002. Etude de la pollution de l'eau souterraine de la ville de Niamey, Niger. Bulletin de la Société de Pathologie Exotique (Bull. Soc. 
Pathol. Exot.), 95(2) : 119-123.

Coulibaly L, Diomandé D, Coulibaly A, Gourène G. 2004. Utilisation des ressources en eaux, assainissement et risques sanitaires dans les quartiers précaires de la commune de Port-Bouët (Abidjan; cote d'ivoire). Vertigo - La Revue en Sciences de l'Environnement, 5(3) : 11.

Davis JC. 1984. Statistics and Data Analysis in Geology (2è edn). Wiley: New-York, Etats-Unis; 550.

Domenico PA, Schwartz FW. 1990. Physical and Chemical Hydrolgeology. Wiley International: New-York, Etats-Unis; 824.

Dongo K, Koffi KF, Brama K, Biémi J, Tanner M, Guéladio C. 2008. Analyse de la situation de l'environnement sanitaire des quartiers défavorisés dans le tissu urbain de Yopougon à Abidjan, Côte d'Ivoire. Vertigo - La Revue en Sciences de l'Environnement, 8(3) : 11.

El Amrani-Paaza N, Benavente J, CruzSanjulian JJ. 1994. Aplicación del análisis de componentes principales al estudio de las caracteristicas físicoquimicas de las aguas del delta rio Adra (Almeria, Espańa). Bol. Geol. Min., 3: 478-484.

El Asslouj J, Kholtei S, El Amrani-Paaza N, Hilali A. 2007. Impact des activités anthropiques sur la qualité des eaux souterraines de la communauté Mzamza (Chaouia, Maroc). Rév. Sci. Eau, 20(3) : 306-321.

Faillat JP. 1990. Origine des nitrates dans les nappes de fissures de la zone tropicale humide - Exemple de la Côte d'Ivoire. $J$. Hydro., 113 : 231-264.

Fofana F. 2005. Evaluation et cartographie de la vulnérabilité à la pollution de la nappe d'Abidjan selon les méthodes Drastic et God. Mém. DEA, Univ. Abobo-Adjamé, Côte d'Ivoire. p. 72.
Granado S, Ettien AA, Boko N, Yao KA. 3, Tanner M, Obrist B. 2006. La vulnérabilité des citadins a Abidjan en relation avec le palu : les risques environnementaux et la monnayabilité agissant à travers le palu sur la vulnérabilité urbaine. La Revue Electronique en Sciences de l'Environnement - Vertigo, Hors Série 3, décembre 2006, 9p. Tropical : la lagune Ebrié (Côte d'Ivoire). Oceanologica Acta, 12(1): 45-55.

Hasley C, Leclerc H. 1993. Microbiologie des Eaux d'Alimentation. Tec \& Doc : Paris.

Hakkou R. 2001. La décharge publique de Marrakech : caractérisation des lixiviats, étude de leur impact sur les ressources en eau et essais de traitement. Thèse de doctorat d'Etat, Université Cadi Ayyad, Marrakech, Maroc, p. 141.

Harris J, Loftis JC. Montgomery RH. 1987. Statistical methods for characterizing ground water quality. Ground Water, 25(2): 185-193.

Herischen D, Ruwaida MS, Blackburn R. 2002. Répondre au défi urbain. Population Reports. Série M, Numéro 16. Info Project. Maryland, USA., p.23.

INS (Institut National de la Statistique) 2001. Recensement Général de la Population et de l'Habitation (RGPH) 1998. Données soci-démographiques et économiques des localités, résultats définitifs par localités, région des lagunes, vol 3, tome 1, 43p.

Jaouad ElA, Sanae K, Namira ElA, Abderrauf H. 2007. Impact des activités anthropiques sur la qualité des eaux souterraines de la communauté Mzamza (Chaouia, Maroc), Rev. Sc. Eau, 20(3) : 309-321.

Jourda JP, Kouamé KJ, Saley MB, Kouadio BH, Oga YS. 2006. Contamination of the Abidjan Aquifer by sewage: An assessment of extent and strategies for protection In Groundwater Pollution in Africa, Yongxin X, Brent U (eds). Taylor 
\& Francis: Balkema, Great-Britain; 293302.

Jourda JP. 1987. Contribution à l'étude géologique et hydrogéologique de la région du Grand Abidjan (Côte d'Ivoire). Thèse Doctorat. Univ. Scient. Techn. et Méd. de Grenoble, p. 319.

Kouadio L., Abdoulaye S., Jourdha J. P., Loba M., Rambaud A. 1998. Conséquences de la pollution urbaine sur la distribution d'eau d'alimentation publique à Abidjan. Cahiers de l'Association Scientifique Européenne pour l'Eau et la Santé, 3(1): 61-75.

Kouamé KJ, Jourda JP, Leblanc Y, Deh SK, Anani AT, Biémi J. 2009. Implication de la modélisation hydrogéologique dans l'estmiation du temps de transfert des polluants vers les eaux souterraines du District d'Abidjan: cas des déchets toxiques. European Journal of Scientific Research, 32(1) : 6-24.

Kouamé KJ. 2007. Contribution à la Gestion Intégrée des Ressources en Eaux (GIRE) du District d'Abidjan (Sud de la Côte d'Ivoire) : Outils d'aide à décision pour la prévention et la protection des eaux souterraines contre la pollution. Thèse de doctorat de l'Université de AbidjanCocody, 225p.

MPD (Ministère du Plan et du Développement). 2006. Rapport national de l'Etat et le devenir de la population de la Côte d'Ivoire (REPCI). Population et Développement: défis et perspectives pour la Côte d'Ivoire. UNFPA.

Mpakan HG, Kamgang KBV, Kouam K, Tamo T, Bemmo N, Ekodeck GE. 2006. L'accès à l'eau potable et à l'assainissement dans les villes des pays en développement: cas de Bafoussam (Cameroun). La Revue Electronique en Sciences de l'Environnement - Vertigo, 17(2) : 10 .

Normes Françaises. 1999. Arrêté № 1639CM fixant les normes de potabilité des eaux destinées à la consommation humaine distribuées par les réseaux, fontaines et citernes à usage collectif.

OMS. 2000. Rapport sur l'évaluation de la situation mondiale de l'approvisionnement en eau et de l'assainissement

OMS. 2007. Prévenir la maladie grâce à un environnement sain, une estimation de la charge de morbidité imputable à l'environnement. Prüss-Üstüm et C. Corvalán, p. 20.

Potvin P, Grimard Y. 1983. Etude de la qualité du milieu aquatique de 67 lacs à sauvagine de la región de Schefferville, Québec. Ministère de l'Environnement, Direction Générale des Inventaires et de la Recherche, Service de la Qualité des Eaux. p. 134.

Poda JN, Gagliardi R, Kam FO, Niameogo A T. 2003. La perception des populations des maladies diarrhéiques au Burkina faso : une piste pour l'éducation aux problèmes de santé. Vertigo - La Revue en Sciences de l'Environnement, 4(1) : 6.

Rodier JC, Bazin, JF, Busby, RW. Lee, Hanshaw BB. 1990. L'Analyse de l'Eau (8è édn). DUNOD : Paris, France.

Savané L, Goula-Bi TA. Aristide DG, Kouamé KL. 2005. Vulnerability assessment of the Abidjan quaternary aquifer using the DRASTIC method. Groundwater Pollution in Africa; 115124.

Siegel S. 1956. Nonparametric Statistics for the Behavioral Sciences. McGraw Hill : New York ; 303.

Soro N. 2003. Gestion des eaux pour les villes africaines, Evaluation rapide des ressources en eau souterraine /occupation des sols (Abidjan), rapport final.

Tomassone R, Dervin C, Masson JP. 1993. Biométrie : Modélisation des Phénomènes Biologiques. Masson: Paris; 1-553.

Traoré A. 2005. Pollution des eaux souterraines de la région d'Abidjan par 
les composes azotes. Bilan des connaissances origine et évolution de ces composés. Mémoire DEA Univ. AboboAdjamé, Côte d'Ivoire, p. 77.

UN-Habitat. 2001. Cities in a Globalizing World: Global Report on Human Settlements. Earthscan: London; 344.

UN-Water/WWAP. 2006. L'eau: une responsabilité partagée. Résumé du $2^{\text {ème }}$ rapport mondial des Nations Unies sur la mise en valeur des ressources en eau. p. 52.
World Health Organization. 2003. Emerging issues in water and infectious disease, 24 p.

World Health Organization, 2008. Guidelines for drinkink-water quality, third edition incorporating the first and second addenda, volume 1, Recommandations, Geneva.

Yonkeu S, Maïga AH, Wethé J, Mampouya M, Maga GP. 2003. Conditions socioéconomiques des populations et risques de maladies: le bassin versant du barrage de Yitenga au Burkina faso. Vertigo - La Revue en Sciences de l'Environnement, 4(1): 15 . 